\title{
LA RESOLUCIÓN DE PROBLEMAS Y LA EDUCACIÓN MATEMÁTICA: HACIA UNA MAYOR INTERRELACIÓN ENTRE INVESTIGACIÓN Y DESARROLLO CURRICULAR
}

\author{
ARRIETA GALIASTEGUI, J.J. \\ Departamento de Ciencias de la Educación. Universidad de Oviedo.
}

\begin{abstract}
SUMMARY
Mathematical Problem Solving is analysed from a position which attempts to study its implications for curricular development in mathematics. The analysis begins by inquiring the assumption of Problem Solving as the axis to develop this work while recognizing its currently relevance and the necessity of an increasing interconnection between research and curricular development in this field. Such interconnection can be seen in the recent works of many researchers and it determines the so-called «postpiagetian paradigm». From the author's perspective, the studies of addition and subtraction problems represent a domain in which this paradigm is well exemplified and from which we can learn a lot to improve both the curriculum and the teacher's training.
\end{abstract}

\section{INTRODUCCIÓN}

La resolución de problemas como eje del desarrollo curricular

Los estudios sobre la resolución de problemas (R.P.) han atraído la atención de los investigadores de los más variados campos en los últimos 50 años. Si nos referimos a la resolución de problemas humanos, no simples, y al estudio de los complejos procesos que en ellos subyacen desde una base de aceptación de la necesidad de ser explícitos sobre los mecanismos internos, simbólicos, que se ponen en juego, olvidándonos por tanto de los enfoques conductistas que tendían a presentarlos meramente como ejemplos de aprendizaje, se suele considerar el año 1956 como una fecha clave para el desarrollo del tema desde una perspectiva cognitiva, relacionada con la teoría del procesamiento de la información (Newel y Simon, 1972) aunque habría que resaltar también, en una perspectiva análoga, los análisis neuropsicológicos de los procesos intelectuales directamente implicados en Ia R.P. realizados por Luria y sus colaboradores en la Unión Soviética desde la década de los cuarenta (Luria y Tsvetkova 1981).

En el campo de la educación matemática, sin embargo, y debido al casi nulo desarrollo material e investigador del mismo, no es hasta la pasada década cuando se comienza a estudiar sistemáticamente la R.P. y sus implicaciones curriculares. En la actualidad la R.P. de matemáticas es ciertamente el tema de moda entre los teóricos y prácticos interesados por la enseñanza de las matemáticas y los siguientes datos avalan su consideración como una "corriente educativa»:

- El N.C.T.M. norteamericano (Consejo Nacional de Profesores de Matemáticas) sitúa como primer f́tem en su lista de recomendaciones para la década de los 80 la idea de que la R.P. debe ser el eje de la matemática escolar, el principal objetivo de la enseñanza de las matemáticas (Shufelt y Smart 1983) y dedica el libro del año 1980 íntegramente al tema (Krulik y Reys 1980).

- La A.T.M. inglesa (Asociación de Profesores de Matemáticas), fundamentándose en el párrafo 249 del informe Cockcroft (1982) que establece que la habilidad en R.P. es el corazón de las matemáticas, elabora un escueto documento en el que se afirma taxativamente que la R.P. podría y debería reemplazar a la aritmética rutinaria como el tema principal en las clases primarias (A.T.M. 1985).

- En las conferencias y congresos sobre educación matemática las ponencias y comunicaciones relacionadas con la R.P. dominan claramente el panorama tras un lento proceso de incorporación a los mismos, desplazando a las relacionadas con la «nueva matemática» o el movimiento de «back to basics». Hay que re- 
cordar que con anterioridad al VI.C.M.E., en los cuatro congresos internacionales previos, apenas se trató el tema con cierta relevancia, aunque ya desde el Il I.C.M.E. se encuentran aportaciones al mismo, como las de Polya (1973) o Biggs (1973), así como con diversos grupos de trabajo que lo abordan específicamente.

- Publicaciones, proyectos y centros de investigación relacionados con la R.P. en matemáticas están apareciendo continuamente desde mediados de la década pasada y con apoyos económicos muy fuertes, destacando los concedidos por el National Institute of Education de los EE UU a diversos institutos de investigación especializados. Como muestra del esfuerzo teórico realizado al respecto basta con citar la amplia bibliografía elaborada por Schoenfeld (1983).

- Los resultados de las encuestas entre profesionales y profesores de matemáticas coinciden en señalar la importancia que se le debe asignar a la R.P. en los currículos de matemáticas. Las conclusiones de diferentes encuestas realizadas en los EE UU al respecto son Io suficientemente explícitas: hay un acuerdo total sobre la necesidad de dar más énfasis a la R.P. en la matemática escolar, hay plena unanimidad sobre los objetivos de la instrucción en R.P. y sobre las estrategias apropiadas para los niveles elementales de escolaridad, se prefiere metodológicamente la utilización de los problemas como medios para desarrollar e introducir los contenidos matemáticos y se establece que la R.P. es importante para todos los alumnos, por lo que se debe introducir desde las edades más tempranas (Osborne y Karsten 1980).

Podemos, por tanto, afirmar con Schoenfeld (1985) que el movimiento a favor de la enseñanza de la R.P. comienza como tal a finales de la década de los 70 , fundamentado pedagógicamente en el rechazo tanto de la "nueva matemática) o "matemática moderna» como al intento de vuelta atrás que supuso el «back to basics». Pronto se comprendió, con respecto a este último movimiento, que dominar lo fundamental no era suficiente, sobre todo si se entendia por tal el énfasis en los ejercicios y en la repetición, el dominio de los algoritmos y las operaciones básicas, pues los alumnos tenian que ser capaces de pensar matemáticamente y de poder resolver problemas complejos. $\mathrm{La}$ atención se enfocó en la R.P. y en la interpretación de la misma como objetivo, como proceso y como destreza básica (Branca, 1980), elaborándose diferentes proyectos curriculares centrados en la R.P.

Como contraste, los alarmantes datos de los diferentes informes internacionales sobre las limitadas capacidades de los estudiantes puestas de manifiesto en el trance de resolver problemas algo complejos, además de servir de acicate a los recientes esfuerzos realizados en las investigaciones sobre el tema, justifican plenamente el acentuado énfasis en su consideración como eje de la matemática escolar.
Sin embargo, la R.P. no es ni puede ser la panacea que resuelva los complejos problemas que encierra la tarea de enseñar matemáticas en la escuela actual. Si no cabe ninguna duda de que constituyen el corazón de las matemáticas, hay que tener presente que los problemas se resuelven eficazmente por medio de la aplicación de la teoría matemática apropiada, de los conocimientos básicos (hechos, destrezas, conceptos, estructuras conceptuales y estrategias generales) necesarios. También es cierto que es precisamente el deseo, el interés por resolver problemas interesantes el que puede provocar el aprecio por la teoría, por los conocimientos, objetivo más difícil de lograr pero de gran valor educativo.

Al igual que la mera construcción de teorias (por ejemplo, las estructuras matemáticas) sin ninguna relación con los problemas a los que pueden aplicarse no es una actividad matemática juiciosa, sino una caricatura del método axiomático, caricatura en la que incurrió el movimiento de la "matemática moderna», la concentración exclusiva en la R.P. supone una deformación de los principios que subyacen en el método de descubrimiento guiado en educación y, sobre todo, una limitación en el logro de objetivos que la matemática, como conocimiento científico, puede aportar a la educación. Las dos actividades, la de construcción de conceptos y estructuras y la de R.P., son altamente complementarias la una de la otra (Hilton 1977). De lo que se trata es de construir el cuerpo de conocimientos abstractos en un contexto de R.P., manteniendo un balance equilibrado entre ambos aspectos complementarios, el instrumental y el teórico abstracto.

Sin embargo, el énfasis en la R.P. tiene en la actualidad razón de ser, a pesar de las anteriores advertencias, por el predominio de una enseñanza marcadamente formalista, resultante del movimiento dominante de reforma en la década de los 60 (y, en nuestro país, en los 70). El profesorado no dispone de un listado suficientemente rico de problemas para abordar los contenidos instructivos $y$, lo que es aún peor, no sabe en muchas ocasiones cómo aplicar las teorías y los conceptos que tienen que explicar. Por otra parte, la ausencia casi total de problemas de búsqueda y de situaciones abiertas de investigación impide que se desarrollen las estrategias heurísticas necesarias para la R.P. reales e interesantes, que son, además, los que mejor muestran el poder de un conocimiento sólido de los conceptos, teorías y estructuras matemáticas.

Por todo ello, un enfoque instructivo amplio y propiamente didáctico de la R.P. que sepa integrarla.en el currículo escolar debe considerar que se pueden y deben emplear actividades de R.P. con objetivos muy diversos. Tanto en la investigación y descubrimiento de los conceptos, como en su desarrollo y en la construcción de estructuras así como en la ejercitación y práctica de las mismas tiene sentido utilizar un enfoque centrado en la R.P. Si los conceptos matemáticos se han ido construyendo a lo largo de la historia como instrumen- 
tos para resolver determinados problemas, ¿por qué en el ámbito educativo no podemos efectuar la trasposición didáctica adecuada para que los estudiantes sientan también su necesidad? Limitar el objetivo de la R.P. al aprendizaje de heuristicas generales y especiales y a la aplicación de conocimientos previamente adquiridos supone desvincularla del proceso de construcción de los conceptos y teorias matemáticas, lo que conduce a considerarla como un añadido para hacer más grata la tarea de aprender matemáticas, como un caramelo que endulza las labores arduas. Y no se trata de eso.

Si se pretende organizar el currículo de matemáticas en torno a la R.P., ésta debe poder jugar un papel en todas y cada una de las actividades del aula de matemáticas. Lo que hay que demostrar es que tal planteamiento es factible y para ello es preciso, como minimo, que se cree en las aulas un ambiente de trabajo en el cual puedan ralizarse efectivamente actividades de R.P. y que se disponga de materiates curriculares apropiados para ello. En este sentido, la evolución paralela de las investigaciones sobre R.P. junto con el desarrollo de proyectos curriculares centrados en la misma presenta unas características claramente prometedoras con vistas al futuro-presente. Como vamos a ver en los próximos apartados, dicha evolución va en la linea de favorecer un planteamiento mucho más interrelacionado entre las investigaciones y el desarrollo curricular.

\section{LAS INVESTIGACIONES Y ESTUDIOS EN R.P: HACIA UN NUEVO PARADIGMA DE INVESTIGACIÓN}

Volviendo la vista atrás, hay que reconocer que la primera llamada seria de atención dirigida a considerar la R.P. como un tema de interés central para el profesorado y el estudiante de matemáticas en los niveles superiores de escolaridad (Bachillerato y Universidad) la constituyó el «How to solve it» de Polya, cuya primera edición data de 1945 (Polya 1982). Más de 10 ediciones del libro en castellano hasta la actualidad garantizan su popularidad y su influencia. Su trabajo se centró especialmente en la utilización de un conjunto bien seleccionado de problemas para fomentar su efectiva resolución y la aplicación de procesos heuristicos generales en la resolución de los mismos. Los estadios que Polya propuso como necesarios para una correcta R.P.: comprensión del problema, concepción de un plan, ejecución del plan y la visión retrospectiva, junto con las preguntas que todo resolutor debe hacerse en cada una de las fases, se convirtieron desde entonces en punto de referencia ineludible de todo trabajo al respecto.

Este simple modelo de lo que ocurre durante la R.P. puede servir, y asi se ha utilizado en numerosas ocasiones, como un esquema para deducir modelos e incluso materiales de instrucción y evaluación. Así, por ejemplo, ŁeBlanc y sus colaboradores (1980) proponen un modelo instructivo para la R.P. en las escuelas elementales basado literalmente en las ideas de Polya. Algo muy similar es lo que propone Fennell (1983), al referirse también a la R.P. en los grados primarios. Otros autores abogan por su utilización para elaborar una guía de R.P. puesta a disposición del alumnado, siempre y cuando éste la necesite, hasta que la vayan interiorizando paulatinamente. Así por ejemplo, Charles y Lester (1982) proponen una guía claramente deducida del modelo de Polya, y especifican al máximo la labor docente a realizar por el profesorado antes, durante y después de la resolución del problema propuesto, indicando diez acciones de enseñanza para la R.P. y el propósito de cada una de ellas. Por su parte, en el ámbito de la evaluación, las aportaciones de Schoen y Oemhke (1980), al desarrollar el Iowa Problem Solving Test, o de nuevo las de Charles y Lester (1982), al presentar un sistema de puntuación para evaluar el trabajo de los estudiantes en Ia R.P., ejemplifican las virtualidades y deficiencias al respecto del modelo de Polya (relativas a la dificultad de evaluar la segunda fase, la de elegir la estrategia adecuada).

En el ámbito más estricto de la investigación, su influencia se deja notar en obras clave como la editada por Goldin y McClintock (1984) en torno al análisis específico de las variables sintácticas, de contenido y de contexto, estructurales y heurísticas que inciden en la dificultad de las tareas matemáticas. Análisis que pone de manifiesto la utilidad del conocimiento y del control de estas variables por parte del profesorado para, por ejemplo, crear problemas de estructura matemática isomórfica variando la sintaxis, el contenido y el contexto (Caldwell 1984).

De la popularidad e influencia de su obra da buena muestra también el hecho de que haya sido traducido ya en 1959 al ruso y sea uno de los escasísimos autores occidentales citado por los estudiosos del aprendizaje y de la enseñanza de las matemáticas en dicho país. En la obra de Menchinskaya y Moro (1975) sobre cuestiones relacionadas con los métodos y la psicologia de la enseñanza de la aritmética en Ios niveles elementales de escolaridad, publicada en 1965, se dedica un capitulo completo al tema de la R.P., proponiendo una serie de reglas que coinciden claramente con las de Polya, aunque las hayan deducido de sus investigaciones en la escuela soviética.

Otra muestra mucho más reciente de su influencia la encontramos en la obra de Papert (1981). Si bien este autor nos presenta en su «Desafío a la mente» una utopía educacional de lo más atrayente, basada en una interpretación rigurosa de la teoria epistemológica y psicológica de Piaget y en las aportaciones de la Inteligencia Artificial, aquí nos interesa destacar el hecho de que las heurísticas generales para la R.P., que según Polya se deben enseñar, se prestan maravillosamente a ser concretadas en su geometria de la tortuga. Según Papert, la mejor manera de explicar a Polya a 
los estudiantes es dejar que aprendan en la computadora dicha geometría pues ésta suministra excelentes oportunidades de practicar heurísticas como, por ejemplo, la de buscar un problema más simple. Si no se ha conseguido hacer arraigar lo suficientemente las ideas de Polya en los sistemas educativos es por la escasez de buenas situaciones en donde los alumnos puedan encontrar e interiorizar modelos simples y convincentes de conocimiento heurístico y de ahí que la propuesta de Papert no vaya en la linea que comentábamos anteriormente, la de utilizar explícitamente el esquema de Polya para elaborar modelos y materiales educativos, sino que lo que nos propone es «poner el consejo de Polya con los pies sobre la tierra». Esto es, nos presenta la geometría de la tortuga como terreno introductorio ideal para el aprendizaje del pensamiento heurístico, para el ejercicio de los principios de Polya, dado que la matemática escolar, aunque elemental en términos de su contenido aritmético, es una materia relativamente avanzada para el ejercicio de los mismos.

No le falta razón en este punto a Papert, como lo muestra por otra parte el hecho de que la mayoría de las aplicaciones del modelo de Polya se hayan referido a niveles superiores de escolaridad, aunque opino, y nuestro trabajo pretende entre otras cosas demostrarlo ${ }^{(1)}$, que minusvalora las posibilidades de ejercitár dichos principios en situaciones de aprendizaje que no exijan el concurso de las potentes computadoras que utilizan el lenguaje LOGO. Con sencillos y asequibles materiales manipulativos se pueden plantear verdaderos problemas que requieran la utilización implicita de la mayoria de las heurísticas citadas por Polya, siempre y cuando se utilicen en un contexto que favorezca la ejercitación de los procesos de razonamiento lógicomatemático y la reflexión sobre los mismos, además de no estar mediatizado por la obsesión de lograr la respuesta correcta al problema planteado.

La obra de Polya tuvo una influencia importante en nuestro pais, especialmente en los trabajos del ilustre pionero que fue Puig Adam. Ya en 1959, al plantear su punto de vista cibernético sobre el problema de los problemas (Puig Adam 1985), criticó la insuficiencia y generalidad de los consejos de Polya: «todo cuanto se llega a sacar de esta metodología clásica de los problemas es una cierta costumbre de trazar, de tender caminos que enlacen la solución buscada a las premisas establecidas en la red más o menos vasta de implicaciones lógicas en que están inmersas. Pero a medida que el campo se ensancha y los puntos de partida y de llegada se alejan de las perspectivas corrientes, estos sabios consejos metodológicos muestran una insuficiencia a su generalidad». Por ello valora mucho más las aportaciones de Polya recogidas en su estudio sobre el razonamiento plausible en matemáticas (Polya 1966), pues en ella el razonamiento implicativo, para el que son útiles los consejos de su «How to solve it», cede su lugar al razonamiento plausible fundado en la inducción, en la analogia, en la inferencia, etc... De to- das formas, en toda su obra didáctica (Puig Adam 1956, 1960) es patente el acuerdo general de sus planteamiento pedagógicos con los de Polya.

Más recientemente, y en nuestro país, se aprecia también la incidencia de la obra de este autor en los trabajos del grupo Cero de Valencia así como en los interesantes estudios de Miguel de Guzmán. En el diseño curricular propuesto para alumnos de 12 a 16 años por el Grupo Cero de Valencia (Grupo Cero 1985) se enfatiza la importancia de las estrategias de la R.P. y de las capacidades básicas que se consolidan mediante la actividad matemática: generalizar, abstraer, hacer hipótesis y someterlas a pruebas, explorar, tomar decisiones, proponer ideas nuevas, hacer frente a situaciones problemáticas con la confianza de que pueden ser comprendidas y, en su caso, resueltas. Por su parte, Miguel de Guzmán (Guzmán 1986), al plantearse el problema de la utilización de juegos en la enseñanza y tras señalar la semejanza de estructura entre el juego y la matemática (de manera similar a como lo hace Gallagher, 1980), presenta un esquema para favorecer el desarrolio heurístico a través de los juegos basado también literalmente en las fases o estadios propuestos por Polya. Los reinterpreta para mostrar la semejanza de actitudes que se dan en la resolución de un puzzle o de un juego y en la de un genuino problema matemático y cómo la mayoría de los hábitos adecuados para la tarea escolar podrian adquirirse con ejemplos escogidos de juegos.

Lo escrito hasta ahora es suficiente para mostrar la persistente y perdurable influencia a lo largo de los años de las iniciales y germinales aportaciones de Georges Polya asi como la popularización de sus ideas en todos los países desarrollados. Sin embargo, los enfoques curriculares centrados en su modelo se han mostrado insuficientes en, al menos, dos ámbitos de actuación: los aspectos metacognitivos de las conductas de R.P. y la aplicación de las destrezas de R.P. a situaciones reales en las que se dispone de una variedad de recursos ajenos (calculadoras, libros, otros estudiantes y profesores asistentes...).

Como afirman Schoenfeld $(1979,1982,1983)$ y Garofalo y Lester (1985), la instrucción en R.P. de matemáticas se ha centrado fundamentalmente hasta la fecha, y con un éxito cuestionable, en las heurísticas o tácticas de resolución. A su juicio, gran parte del escaso exito de los enfoques anteriores reside en el hecho de que las conductas directivas o estratégicas han sido demasiado ignoradas. Schoenfeld entiende por ello las decisiones que tienen un impacto central en la dirección de búsqueda de la solución y en la localización de los propios recursos durante el proceso de R.P. y su argumentación no puede ser más contundente: incluso si tenemos éxito al adiestrar a nuestros alumnos en la aplicación de cada una de las estrategias heuristicas, no podemos esperar un rendimiento mejor de los mismos cuarido los evaluemos utilizando una amplia variedad de problemas que requieran distintas heurísti- 
cas. Deben ser capaces de seleccionar los medios ade. cuados para cada problema y si carecen de esa habilidad no pueden obtener beneficios de sus recursos heurísticos. Por ello, los currículos orientados «metacognitivamente» hacen mucho hincapié en la discusión entre estudiantes, en el pensamiento sobre los procesos de resolución y, en general, en la toma de conciencia de los mismos procesos.

La segunda insuficiencia que se pone de manifiesto al revisar los estudios sobre la enseñanza de las estrate. gias heurísticas generales o especiales se refiere a su aplicacion en situaciones reales. Las aportaciones de Lesh y sus colaboradores $(1980,1982,1983,1985)$ han sido decisivas al respecto. Los dos proyectos que han desarrollado hasta la fecha, el Applied Problem Solving (A.P.S.) y el Rational Number Project (R.N.), son inusuales entre los relacionados con la R.P. porque sur. gen de sus investigaciones sobre la formación de conceptos, en vez de partir del desarrollo de un enfoque instructivo o de la investigación sobre R.P. propiamente dicha. Esto es, pretenden trazar el desarrollo de determinadas ideas matemáticas e identificar características de las tareas que influyen en la habilidad para utilizarlas en situaciones particulares. Un intento central del A.P.S. es el de procurar responder a la siguiente pregunta: más allá del hecho de «tener» una idea, ¿qué es lo que le permite a un estudiante promedio utilizarla en situaciones de la vida cotidiana? Para ello parten de la base que los procesos más importantes de R.P. aplicados contribuyen significativamente tanto a la comprensión como a la utilización de las ideas matemáticas. No es necesario que los estudiantes aprendan primero una idea, luego algunos procesos generales de R.P. (como asumen, implícitamente, los anteriores enfoques) y, finalmente, utilicen la idea y los procesos en situaciones reales. Más bien se da una interacción dinámica entre el contenido de las ideas matemáticas y los procesos empleados para resolver problemas basándose en esas ideas.

Una hipótesis como la anterior, que asumimos plenamente, tiene implicaciones curriculares determinantes. En primer lugar, porque es muy poco probable que las aplicaciones y la R.P. sean bien aceptadas en las escuelas, a menos que el profesorado se convenza de que juegan un papel destacado en la adquisición de ideas matemáticas, y de que pueden y deberían utilizarse como un contexto en el que tenga lugar el aprendizaje de conceptos matemáticos. En segundo lugar, porque dado que los procesos, destrezas y comprensiones ne. cesarias en las aplicaciones de las matemáticas en la vida cotidiana no son necesariamente las mismas que las destacadas por los lideres del movimiento a favor de la R.P. en las escuelas (Lesh y Akestrom 1982), un énfasis adecuado en la enseñanza de las aplicaciones de las matemáticas puede requerir que algunos tópicos se in. serten en el currículo, mientras que otros se deben re tirar, En tercer lugar, porque como los problemas de los textos difieren significativamente de sus imágenes en el mundo real, tanto con respecto a su grado de dificultad como en los procesos necesarios para su resolución y en los tipos de errores cometidos con mayor frecuencia, es preciso desplazar el énfasis desde los procesos generales (pero débiles) independientes del contenido hacia los poderosos procesos de resolución relacionados con este contenido. Por último, y en cuar* to lugar, porque la interpretación de situaciones reales, al utilizar ideas matemáticas, constituye una gran parte de lo que se entiende por comprensión matemática y la creación, modificación y adaptación de los mo. delos empleados para filtrar, organizar e interpretar la información que sea más útil para encontrar una respuesta lo suficientemente buena son parte importante de la R.P. así como también la formación de conceptos. El cultivo de estas destrezas deberia constituir un eje prioritatio de los currículos de matemáticas del futuro.

En un sentido similar, se han planteado críticas al mo* delo de Polya y a sus implicaciones curriculares por no proporcionar una visión del proceso de resolución de problemas que esté de acuerdo con la metodología científica (Gil y Torregrosa 1983). A juicio de estos autores, su posición manifiesta una interpretación excesivamente simplista y positivista del proceso de producción del conocimiento científico, desconsiderando el papel de las hipótesis y teorias en las que encajan los «datos» del problema asi como el propio proceso de buscar y plantearse problemas.

La misma evolución interna en el ámbito de la investigación sobre R.P. ha servido también para fundamen. tar y apoyar las anteriores críticas, pues ellas se desprenden casi linealmente de sus características, como vamos a ver a continuación. Ya decíamos al comienzo que no ha sido hasta finales de los 70 cuando han co. menzado a desarrollarse de manera sistemática las investigaciones sobre R.P. matemáticos, con planteamientos comunes y mejor coordinados. Las anteriores investigaciones no fueron conducidas sistemáticamente dentro de la comunidad de educadores matemáticos debido a la falta de acuerdo, a la inexistencia de un tronco común referido a aspectos como:

- lo que constituye la R.P.

- cómo debe medirse el rendimiento en la R.P.

- qué tareas y métodos son apropiados para los propósitos de la investigación

- cuáles son las variables clave que influyen en las conductas de R.P.

Con ello no quiero decir que recientemente se haya llegado a un acuerdo pleno sobre todas esas cuestiones, pero sí constatar que existen subáreas en el amplio campo de la investigación sobre la adquisición de conceptos y procesos matemáticos que han madurado lo su. ficiente como para que sus perspectivas teóricas y metodológicas no se tomen prestadas sin más de otros campos, especialmente de la psicología evolutiva y de la teoría del procesamiento de la información, sino que 
están planteando el desarrollo de metodologías de investigacion propias, que integran alguna forma de intervención educativa en el proceso de recogida de datos, así como la construcción de teorías específicas relacionadas con la mejora en calidad de la educación matemática.

Para ello ha sido preciso que emergiese un paradigma alternativo en el estudio de los procesos de pensamiento sobre contenidos matemáticos que no esté centrado ni en la enseñanza ni en el aprendizaje, sino en el estudio de los procesos de pensamiento desarrollados ante los problemas matemáticos (Davis 1983). Dentro de este nuevo marco conceptual, el aprendizaje se entiende y se define en términos de transición de una forma de pensamiento matemático a otra y, en este sentido, no es fundamental; es un tipo de concepto derivado o de segundo orden pues la tarea esencial consiste en la descripción de las diversas formas de pensamiento matemático, las que se dan antes y después de cualquier cambio debido al aprendizaje.

Como lo que se quiere investigar son los mismos procesos de pensamiento matemático, los estudios se centran en la observación y descripción de las conductas matemáticas (reconociendo la necesidad de relacionar dichas observaciones con una teoría postulada de los procesos para tratar con la información), y en la recogida de datos (a través, sobre todo, de las entrevistas basadas en tareas). Como vemos, un cambio de orientación que se encuadra perfectamente en el más general que afecta a las investigaciones educativas y que las sitúa en un plano más próximo al que tiene interés para los profesores experimentados, pues a éstos lo que les interesa son, precisamente, los procedimientos que sus alumnos individuales utilizan al analizar y resolver los diferentes problemas matemáticos que se les plantean o que ellos mismos identifican.

Por otro lado, y como afirman Lesh y Landau (1983), en las investigaciones actuales sobre R.P. se está adoptando un tipo de análisis intermedio entre los de tareas muy especificas y aisladas, sin relación con ningún contenido matemático importante, y los de las características cognitivas generales de los niños que se suelen utilizar en las investigaciones psicológicas. Estas últimas tienden a centrarse en las capacidades cognitivas invariantes y generales que se pueden aplicar a diferentes materias o en los cambios antes y después de las reorganizaciones cognitivas más importantes (los estadios piagetianos, por ejemplo) o, por último, en las ideas que los estudiantes adquieren naturalmente sin necesidad de una intervención didáctica especifica. Por otra parte, los análisis de tareas, aunque posibilitan la construcción de modelos explicativos que son suficientes para simular las conductas de los estudiantes en una tarea aislada, no son suficientes para explicar cómo una idea determinada influye en la interpretación y solución de un problema.

Sin embargo, las investigaciones en educación mate- mática dirigidas a trazar el desarrollo de una idea se suelen situar en un punto equidistante de los dos tipos de análisis anteriores, centrándose en las ideas que se adquieren en los niveles intermedios entre los estadios o en los factores y procesos que producen y facilitan la transición de un nivel de conceptualización a otro para algún concepto individual o, por último, en los procesos y capaciđades que están ligados a la comprensión del contenido y en las ideas que no se desarrollan naturalmente sin experiencias instructivas que faciliten su adquisición. Por lo tanto, la clase de procesos que están apareciendo como más importantes en la R.P. matemáticos, al igual que en otros dominios de la R.P., no son las heurísticas generales avanzadas por Polya sino los procedimientos y técnicas relacionados específicamente con contenidos matemáticos: los primeros conceptos numéricos y las primeras operaciones, las estructuras aditivas y multiplicativas, el razonamiento proporcional, los conceptos geometricos, etc...

El mismo tipo de técnicas de análisis de ideas se aplica en la actuaifdad incluso al estudio de los procesos metacognitivos, como lo muestran los conocidos trabajos de Schoenfeld (1983), el cual procura ir más allá de la investigación sobre la influencia y el aprendizaje de las diversas heurísticas y estrategias, para estudiar los aspectos de control y de dirección, de decisión ejecutiva, que son necesarios para determinar cuándo pueden ser útiles dichos procesos, cómo pueden relacionarse con otros procesos, etc...

Investigaciones que ponen de manifiesto lo que acabamos de afirmar y que nos pueden servir por tanto para conocer los estudios más interesantes sobre la R.P. matemáticos aparecen recogidos en los libros editados por Ginsburg (1983) y por Lesh y Landau (1983), así como en el más específico, relacionado con la adquisición de la adición y la sustracción desde una perspectiva cognitiva, de Carpenter, Moser y Romberg (1982). Todos ellos son representativos de una orientación que podríamos llamar postpiagetiana (para diferenciarla de la corriente neopiagetiana desarrollada en torno a los trabajos de Case y Pascual Leone y sus colaboradores), puesto que han dejado de lado los intentos de validar las tareas piagetianas y su teoría de los estadios para centrarse en el desarrolio de investigaciones directamente relacionadas con los contenidos escolares (conceptos y procesos matemáticos en este caso) y con una perspectiva mucho más educativa, considerando las prácticas instructivas como una variable más a considerar.

Aunque ya no exista una única teoria dominante, como ocurrió en el pasado con la de Piaget, en los trabajos recogidos en dichos textos es posible identificar dos orientaciones o enfoques de investigación diferentes que se dejan traslucir en función de los diferentes objétivos y puntos de partida que asumen, aunque compartan los rasgos comunes que señalábamos previamente. Además, y curiosamente, estos dos enfoques, el de los profesores de matemáticas y el de los psicólogos cog- 
nitivos, se plasman por separado en los dos textos que citamos en primer lugar.

En efecto, el de Lesh y Landau recoge investigaciones realizadas por profesores de matemáticas que pueden caracterizarse por su inquietud e interés por la variabilidad. Es decir, manifiestan un especial interés por las tareas y las variables de contexto que afectan potencialmente el rendimiento en Ia R.P. y por la varia* bilidad de las conductas. Incluyen, por lo tanto, análisis detallados de tareas e ideas que subyacen en las variables matemáticas y contextuales que influyen en los rendimientos y numerosos datos que describen las conductas de los niños en un conjunto bien definido de tareas. Dan prioridad a la situación de las tareas dentro de un contexto matemático o instructivo y sugieren hipótesis o leyes que gobieman su pensamiento y su rendimiento en las tareas tras presentar los datos sobre cómo los niños las abordan. Hipótesis y leyes que generalmente son muy especificas, directamente relacionadas con las tareas en cuestión y muy apegadas a los datos obtenidos.

En contraste, en el libro cditado por Ginsburg se recogen investigaciones realizadas por especialistas en psicologia cognitiva que siguen una orientación que se podria caracterizar por la importancia que asignan a las similitudes, tanto en las tareas como en los individuos. En vez de centrarse en la variabilidad en los rendimientos, como los anteriores, destacan los rasgos comunes presentando modelos hipotéticos del rendimiento que precisan y simulan los resultados de los esfuerzos de los alumnos en una tarea dada. De esta forma constituyen teorías en pequeña escala sobre cómo actúan ante un conjunto limitado de tareas que representan con. ceptos matemáticos simples o elementales, que pueden modelarse con la suficiente precisión.

Se puede pensar en ambos enfoques como pertenecientes a un mismo programa de investigación, utilizando la terminología de Lakatos (1983), puesto que los dos están de acuerdo en aspectos esenciales: aceptan el mis-

\section{Notas}

(1) Trabajo recogido en la tesis doctoral «Teoría y práctica de las matemáticas en el ciclo inicial de la $E G B$ " que presentamos en el Departamento de Ciencias de la Educación de la Universidad de Oviedo, en junio de 1987; tesis dirigida por el profesor José Gimeno Sacristán, catedrático de Didáctica General en la Universidad de Valencia. mo tipo de datos, extraídos de los protocolos de las entrevistas individuales, y recurren a teorias locales que se construyen de abajo-arriba, en la línea de las deducidas del procesamiento de la información y en contraste con la teoría piagetiana. Ambos enfoques, adcmás, son esenciales para el desarrollo de un programa de investigación que aborde de manera sistemática cl estudio de los procesos de pensamiento matemático que se van construyendo conforme se resuelven problemas y tareas escolares, puesto que las debilidades de cada uno de ellos se ven compensadas con las virtudes del enfoque alternativo y puesto que es posible concretar, al abordar problemas comunes desde las dos perspectivas, aportaciones de gran valor para la didáctica de las matemáticas.

Uno de estos campos donde la colaboración es más estrecha es precisamente el de la adquisición de los conceptos de adición y sustracción. Los trabajos de Carpenter y Moser $(1983,1984)$ así como los de Fuson (1983) y Resnick (1983) ejemplifican perfectamente las posiciones de ambos enfoques y aportan un conjunto de hipótesis y recursos de innegable valor para la fundamentación de proyectos curriculares del área de matemáticas en los niveles iniciales de escolaridad. Sus estudios sugieren aiternativas para secuenciar la enseñanza y medios para evaluar el progreso de los alumnos. Ahora bien, no se trata de incluir los procedimientos que nos muestran como contenidos del currículo; no es cuestión de ir incorporando los descubrimientos psicológicos como tales, como se pretendió hacer con las tareas piagetianas (2). Se trata más bien de incorporarlos como guias que ayudan a programar las actividades escolares con la suficiente variedad de medios $y$ contextos en los que se puedan poner de manifiesto de manera implícita, de manera que se favorezca su desarrollo. Guías que nos son útiles también para adecuar los objetivos educativos al nivel de desarrollo de nuestros alumnos y para evaluarlos teniendo criterios, de manera que esta variable didáctica asuma una función realmente educativa.
(2) Esta y otras críticas las desarrollo detenidamente en las dos comunicaciones que presenté en el Simposio sobre Psicología del Aprendizaje y Desarrollo Curricular realizado en Oviedo, en septiembre de 1986. En ellas se cuestionan determinadas opciones curriculares asumidas por autoras como Constance Kamii o Rheta DeVries, referidas a la «enseñanza» del número en los niveles iniciales de escolaridad. 


\section{REFERENCIAS BIBLIOGRÁFICAS}

ARRIETA, J., 1986. La teoria de Piaget y el desarrollo curricular en matemáticas: a) de la estructura a las funciones; b) de las acciones a las operaciones, Actas del I Simposio sobre Psicología del Aprendizaje y Desarrollo $\mathrm{Cu}$ rricular. (Dirección Provincial de Educación: Oviedo).

A.T.M., 1985. A.T.M. at Westminter, Mathematics Teaching, 111, pp. 44-46.

BIGGS, E., 1973. Investigation and Problem-Solving in Mathematical Education, en Howson, A.G. (ed.), Developments in Mathematical Education. (Cambridge University Press), pp. 213-221.

BRANCA, N.A., 1980. Problem Solving as a Goal, Process, and Basic Skill, en Krulik, S. Reys, R.E. (eds.). Problem Solving in Shool Mathematics (1480 Yearbook). N.C.T.M: Reston, Virginia, pp. 3-8.

CALDWELL, J.H., 1984. Sintax, Content and Context Variabies in Instruction, en Goldin, G.A. y Mcclintock, C.E. (eds.). Task Variables in Mathematical Problem-Solving. (The Franklin Institute Press: Philadelphia), pp. 379-414.

CARPENTER, T.P., MOSER, I.M. y ROMBERG, Th.A., 1982. Addition and Subtraction, a Cognitive Perspective. (Lawrence Erlbaum: Hilisdale, N.J.).

CHARLES, R. y LESTER, F., 1982. Teaching Problem Solving. What, Why \& How. (Dale Seymour Publications: Palo Alto, Ca.).

DAVIS, R.B., 1983. Complex Mathematical Cognition, en Ginsburg, H.P. (ed.). The Development of Mathematical Thinking. (Academic Press: New York) pp. 253-290.

FENNELI., F.S., 1983. Focusing on Problem Solving in the Primary Grades, en Shufelt, G. y Smart, J.R. (eds.), The Agenda in Action (1983 Yearbook). (N.C.T.M.: Reston, Virginia), pp. 33-41.

FLAWEI.L, J.H., 1976. Metacognitifs Aspects of Problem Solving. En Resnick, L.B. (ed.). The Nature of Intelligence. (Lawrence Erlbaum: Hillsdale, N.J.).

GALLAGHER, K., 1980. Problem Solving through recreational Mathematics, en Krulik, S. y Reys, R.E. (eds.), Problem Solving in School Mathematics (1980 Yearbook). (N.C.T.M. Reston, Virginia), pp. 169-178.

GAROFALO, J. y LESTER, F.K., 1985. Metacognition, Cognitive Monitoring and Mathematical Performance. Journal for Research in Mathematics Education. Vol. 16 (3), pp. 163-176.

GII. PÉREZ, D. y MARTÍNEZ TORREGROSA, J., 1983. A model for Problem Solving in accordance with scientific methodology. European Journal for Science Education, Vol. 5, (4), pp. 447-455.

GINSBURG, H.P. (ed.), 1983. The Development of Mathematical Thinking. (Academic Press: New York).

GOLDIN, G.A. y McCLINTOCK, C.E. (eds.), 1984. Task Variables in Mathematical Problem-Solving. (The Franklin Institute Press: Philadelphia).

GRUPO CERO DE VALENCIA, 1984. De 12 a 16. Un proyecto de curriculum de motemáticas. (Nau Llibres: Valencia).

GUZMÁN, M., 1986. Aventuras Matemáticas. (Labor: Madrid).
HIL.TON, P., 1977. Education in Mathematics and Science Today. The Spread of False Dichotomies, en Athen, $\mathrm{H}$. y Kunle, H. (eds.). Proceedings of the Third International Congress on Mathematical Education. (Zentralblatt für Didaktik der Mathematic: Karlsruhe), pp. 75-97.

HOLLOWAY, C. et al., 1978. Cognitive Psychology. Learning and Problem Solving ( 3 vols.). (The Open University Press: Waiton Hall).

KRULIK, S. y REYS, S.E. (eds), 1980. Problem Solving in School Mathematics (1980 Yearbook). (N.C.T.M: Reston, Virginia).

KULM, G., 1984. The classification of Problem Solving research variables, en Goldin, G.A. y McClintock, C.E. (eds), Task Variables in Mathematical Problem-Solving. (The Franklin Institute Press: Philadelphia), pp. 1-22.

LE BLANC, J.F., PROUDFIT, L. y PUTT, I.J., 1980. Teaching Problem Solving in the Elementary School, en Krulik, S. y Reys, R.E. (eds.), Problem Solving in School Mathematics (1980 Yearbook) (N.C.T.M: Reston, Virginia), pp. 104-117.

LAKATOS, I., 1983. La metodología de los programas de investigación cientifica. (Alianza: Madrid).

LESH, R. y AKERSTROM, M., 1982. Applied Problem Solving: Priorities for Mathematics Education Research, on Lester, F.K. y Garofalo, J. (eds.), Mathematical Problem Solving: Issues in Research. (Franklin Institute Press: Philadelphia), pp. 110-122.

LESH, R. y LANDAU, M. (eds.), 1983. Acquisition of Mathematics Concepts and Processes. (Academic Press: London).

LESH, R. LANDAU, M. y HAMILTON, E., 1983. Conceptual models and Applied Mathematical Problem Solving Research, en Lesh, R. y Landau, M. (eds.), Acquisition of Mathematics Concepts and Processes. (Academic Press: London), pp. 263-343.

LESTER, F.K., 1983. Trends and Issues in Mathematical Problem Solving Research, en Lesh, R. y Landau, M. (eds.), Acquisition of Mathematics Concepts and Processes. (Academic Press: London) pp. 229-261.

LESTER, F.K. y GAROFALO, J. (eds.), 1982. Mathematcal Problem Solving: Issues in Research. (Franklin Institute Press: Philadelphia).

LURIA, A.R. y TSVETKOVA, 1981. La resolución de problemas y sus trastornos. (Fontanella: Barcelona).

MASON, J, BURTON, L. y STACEY, K., 1985. Thinking Mathematically. (Addison Weslcy: Wokingham).

MEC, 1985. La enseñanza de la matemática a debate (Subdirección General del Perfeccionamiento del profesorado: Madrid).

MEC, 1985. Didáctica de las Matemáticas. Nueva Revista de Enseñanzas Medias, 7.

MENCHINSKAYA, N.A. y MORO, M.I., 1979. Teaching arithmetic in the elementary school, en Kilpatrick, J. y Wirszup, I. (eds.), Soviet Studies in the Psychology of Learning and Teaching Mathematics, Vol. XIV. (N.C.T.M.: Reston). 
NELSON, D. y KIRPATRICK, J, 1975. Problem Solving, en Payne, J. (ed.), Mathematics learning in early childhood. (N.C.T.M.: Reston, Virginia), pp. 69-94.

NEWELL, A., SIMON, H.A., 1972. Human Problem Solving. (Prentice Hall: Londres).

OSBORNE, A. y KASTEN, M.B., 1980. Opinions about Problem Solving in the Curriculum for the 1980's, en Krukil, S. y Reys, R.E. (eds.), Problem Solving in School Mathematics (1980 Yearbook). (N.C.T.M.: Reston, Virginia), pp. $51-60$.

PAPERT, S., 1981. Desafio a la mente. Computadoras y educación. (Galápago: Buenos Aires).

POLYA, G., 1966. Matemáticas y razonamiento plausible. (Tecnos: Madrid).

POLYA, G., 1973. As I read them, en Howson, A.G. (ed.), Developments in Mathematical Education. (Cambridge University Press), pp. 77-78.

POLYA, G., 1982. Cómo plantear y resolver problemas. (Trillas: México), (10 ed.).

PUIG ADAM, P., 1985. Ensenanza heuristica de la matemática. Nueva Revista de Enseñanzas Medias, 7, pp. 23-37.

PUIG ADAM, P., 1985. Un punto de vista cibernético sobre el problema de los problemas. Nueva Revista de Enseñanzas Medias, 7, pp. 38-41.

RESNICK, L.B. y GLASER, R., 1976. Problem Solving and Intelligence. (John Wiley \& Sons: New York).

SCHOEN, H.L. y OEHMKE, Th., 1980. A new approach to the measurement of problem solving skills. En Krulik, S. y Reys, R.E. (eds.), Problem Solving in School Mathematics (1980 Yearbook). (N.C.T.M.: Reston, Virginia), pp. 216-227.

SCHOENFELD, A.H., 1979. Can heuristics be taught?, en Lochhead, J. y Clement, J. (eds.). Cognitive process Instruction. (The Franklin Institute Press: Philadelphia), pp. $315-338$.

SCHOENFELD, A.H., 1982. Some thoughts on problem solving research and mathematics education, en Lester, F.K. y Garofalo, J. (eds.), Mathematical Problem Solving: Issues in Research. (Franklin Institure Press: Philadclphia), pp. 25-35.

SCHOENFELD, A.H., 1983. Problem Solving in the mathematics curriculum: a report, recommendations, and an annotated bibliography. (The Mathematical Association of America. Committee on the Teaching of Undergraduate Mathematics: Wastington).

SCHOENFELD, A.H., 1985. Ideas y tendencias en la resolución de problemas, en $\mathrm{MEC}$, La enseñanza de la mate. mática a debate. (Subdirección General del Perfeccionamiento del Profesorado: Madrid), pp. 25-30.

SHUFELT, G. y SMART, J.R. (eds.), 1983. The Agenda in Action (1983 Yearbook). (N.C.T.M.: Reston, Virginia).

SILVER, E.A., BRANCA, N.A. y ADAMS, V.M., 1980. Metacognition: the missing link in Problem Solving, en Karplus, R. (ed.), Proceedings of the Fourth International Conference for the Psychology of Mathematics Education. (Lawrence Hall of Science: Berkeley), pp. 213-221. 